\title{
Las relaciones entre los sentidos de la actividad de estudio y el pensamiento teórico
}

\author{
As relações entre os sentidos da atividade de estudo e o pensamento teórico \\ Relationships between the senses of the study activity and theoretical thought
}

\author{
Rafael García Cañedo ${ }^{2}$ \\ Eliéte Zanelato ${ }^{3}$ \\ Sônia da Cunha Urt ${ }^{4}$
}

\begin{abstract}
Resumen: En este trabajo nos proponemos desde el enfoque histórico-cultural, realizar un estudio teórico acerca dos sentidos de la actividad de estudio, especialmente en las tareas escolares propuestas pelos profesores, en intuito de posibilitar el desarrollo del pensamiento teórico. En el transcurso del texto nos dedicamos a resolver el siguiente problema: ¿Qué son los sentidos de la actividad de estudio? ¿ejercen influencia en la ejecución de las tareas pedagógicas? En la búsqueda de respuestas traemos los estudios de Leontiev (1978; 2004), Davidov (1978), Davidov y Slobódchikov (1991). Evidenciamos que las formas como el profesor propone las tareas son importantes para la formación de motivos para la actividad de estudio, de sentidos y de desarrollo del pensamiento teórico.
\end{abstract}

Palabras clave: Actividad de estudio. Sentidos. Pensamiento teórico.

Resumo: Neste trabalho nos propomos, a partir do enfoque histórico-cultural, realizar um estudo teórico acerca dos sentidos da atividade de estudo, especialmente nas tarefas escolares propostas pelos professores, no intuito de possibilitar o desenvolvimento do pensamento teórico. No transcurso do texto nos dedicamos a resolver as seguintes questões: O que são os sentidos da atividade de estudo? Eles exercem influência na execução das tarefas pedagógicas? Na busca por respostas trazemos os estudos de Leontiev (1978; 2004), Davidov (1978), Davidov e Slobódchikov (1991)). Evidenciamos que as formas como o professor propõe tarefas são importantes para a formação de motivos para a atividade de estudo, de sentidos e de desenvolvimento do pensamento teórico.

Palavras-chave: Atividade de estudo. Sentidos. Pensamento teórico.

Abstract: In this work we propose, based on the historical-cultural approach, to carry out a theoretical study about the meanings of the study activity, especially in the school tasks proposed by the teachers, in order to enable the development of theoretical thinking. In the course of the text we are dedicated to solve the following problem: What are the meanings of the activity of study? Do they influence the performance of pedagogical tasks? In the search for answers we bring the studies of Leontiev (1978; 2004), Davidov (1978), Davidov and Slobódchikov (1991). We show that the ways in which the teacher proposes the school tasks are important for the formation of motives that drive the activity of study, senses and the development of theoretical thinking.

Keywords: Study activity. Senses. Theoretical thinking.

\footnotetext{
${ }^{1}$ Enviado el: 22 mar. 2019 - Aceptado el: 27 abr. 2019 - Publicado el: 22 oct. 2020

2 Universidad Tecnológica de La Habana "José Antonio Echeverría" (CUJAE) - Email: rafaelgarciacanedo@gmail.com

${ }^{3}$ Universidade Federal de Rondônia (UNIR) - Email: elietezan@gmail.com

${ }^{4}$ Universidade Federal de Mato Grosso do Sul (UFMS) - Email: surt@terra.com.br
} 


\section{Introducción}

Este trabajo es el resultado de diálogos establecidos entre nosotros, los autores de ese artículo, ya profesores y involucrados en el proceso de doctorado, en Brasil y en Cuba, todos con la misma base teórica, la teoría histórico-cultural. Esta teoría tuvo como principal proponente Vigotski (1896-1934) y el objetivo era crear una psicología materialista para comprender el ser humano en su totalidad. Se unieron a Vigotski inicialmente A. Luria (1902-1977) y A. N. Leontiev (1903-1979) formando lo que algunos llamaron troika y, posteriormente, varios continuadores, entre ellos V. Davidov (1930-1998).

Mediante el aparato teórico construido por los precursores de la teoría históricocultural, en el presente texto nos guiaremos en Leontiev ${ }^{\mathrm{i}}$ y Davidovii por creer que con sus estudios es posible reflejar las tareas pedagógicas de los profesores para que favorezcan el aprendizaje de los contenidos producidos por la humanidad durante el proceso de actividad de estudios.

Conocer el sentido que tiene el sujeto de la actividad que realiza es vital para conocer el móvil que la motiva, la fuente impulsora y energizadora de su comportamiento, de sus actitudes y poder predecir los pasos que marcan el camino presente y futuro del sujeto. Se puede propiciar su desarrollo apoyarlo con recursos que le ayuden a seguir adelante, incluso en los momentos más difíciles de su vida.

Es básico entonces, si se quiere guiar el comportamiento y la actitud hacia el estudio de los escolares, investigar y determinar en ellos el sentido de su actividad de estudio. Esto permitiría conocer los móviles que los guían y influir en ellos, ayudarlos a modificarlos en caso de que están herrados, inducirles la formación de nuevos sentidos que los apoyen y los impulsen en su proceso de aprendizaje, y los motiven a estudiar.

Según sean las distintas situaciones sociales del desarrollo en que se encuentren los estudiantes se configurarán la formaciones psicológicas denominadas sentidos de la actividad de estudio y, según como construyan por los estudiantes, su actividad será cierta, verdadera, auténtica y coherente con el lugar en que se encuentran, con el espacio y contexto donde están, y contribuirán con sus aportes al proceso de enseñanza aprendizaje, o estarán fuera de lugar, descontextualizados y descolocados con conductas disfuncionales y saboteadoras en la escuela.

Si los estudiantes realizan su actividad de estudio motivados para aprender y comprender lo que se les enseña, si tienen bien interiorizado la importancia que tiene lo que aprenden para su futuro y tienen conciencia del valor de su esfuerzo para su desarrollo personal realizarán una actividad de estudio verdadera. De lo contrario será un simple acto de estudio que no los guiará a la búsqueda de las esencias ni al desarrollo de su pensamiento teórico, sino que se conducirán con conductas y estrategias superficiales como las de querer aprobar o estudiar por obligación, y no gozarán del placer del aprendizaje real y desarrollador.

Partimos del supuesto que la actividad de estudio influye en la formación del pensamiento teórico, motivando la argumentación de ideas del estudiante frente a los colegas 
y su posicionamiento frente a los argumentos de los demás. Para ello, es dependiente de la cultura del lenguaje ya adquirida.

Es por lo planteado que, en este trabajo, nos proponemos desde el enfoque histórico cultural:

- Hacer un estudio teórico sobre el sentido de la actividad de estudio en los escolares a partir de estudios realizados por Leontiev $(1978 ; 2004)$ y estudio teórico sobre o desarrollo del pensamiento teórico por Davidov (1978), Davidov y Slobódchikov (1991).

- Demostrar la importancia de este conocimiento como un recurso necesario que debe ser utilizado como estrategia por el maestro en su labor diario en el aula para impulsar y propiciar el desarrollo del estudiante a fin de perfeccionar el trabajo del docente para que cumpla con su función como educador de las futuras generaciones.

- Hacer recomendaciones a los educadores para aplicar estos conocimientos.

- Resolver el siguiente problema: ¿Qué son los sentidos de la actividad de estudio? ¿Ejercen influencia en la ejecución de las tareas pedagógicas?

\section{Los sentidos y el desarrollo del pensamiento teórico}

Al actuar en el medio en que vive, el ser humano modifica la naturaleza (externo) y modifica también sus funciones psíquicas (interno). A diferencia de otros animales, el ser humano es capaz de realizar una actividad orientada hacia un determinado fin, tal actividad posibilita una mediación entre el individuo y la realidad objetiva.

De acuerdo con Leontiev (1978; 2004), la realización de actividades promueve el desarrollo psíquico de los individuos y en determinados períodos de la vida unas son más determinantes que otras, son las llamadas actividades rectoras, a saber: el juego en la infancia, el estudio en la edad escolar y el trabajo a partir de la juventud. El cambio de la actividad rectora no debe ser comprendido de manera estanca, es un proceso y como tal, ocurre gradualmente ante las relaciones contradictorias que se establecen.

La estructura de esta actividad, según Leontiev (1978; 2004), está constituida por: necesidades y motivos, acciones, operaciones y objeto (fines). En el proceso de desarrollo el individuo modifica la naturaleza para satisfacer sus necesidades, a medida que éstas son satisfechas, las relaciones sociales se complejizan y surgen nuevas necesidades. Las necesidades se transforman en motivos de la actividad, es decir, impulsan la actividad. Leontiev (1978) señala que existen necesidades ligadas a las cuestiones biológicas y existen las necesidades objetivas que tienen la función de orientar y regular la actividad concreta del sujeto en el medio objetivo. Las necesidades objetivas son aquellas adquiridas a partir de la práctica social, son ellas que estimulan y dirigen la actividad.

Conforme a Leontiev (1978; 2004), la dinámica entre el motivo (impulsor) y el objeto de la actividad humana (final) está constituido por un punto fundamental y genérico: las relaciones sociales. Ellas están dialécticamente ligando motivo y objeto de la actividad del individuo, envueltas de dinámicas como la realización de acciones no lineales. El motivo no 
está siempre conectado inmediatamente a su fin. Para la satisfacción de una necesidad, el individuo constantemente ejecuta acciones y operaciones que no contacten directamente con el fin / objeto de la actividad.

La actividad se constituye de acciones que, a su vez, son ejecutadas por operaciones, evidenciando un distanciamiento entre motivo y fin, es decir, "[...] la separación entre el motivo y el objeto de la actividad individual es resultado de la parcelación en las diferentes operaciones de una actividad compleja [...]" (LEONTIEV, 2004, p. 83). El individuo realiza una cadena de acciones - no aditivas - en respuesta a una tarea, a un fin que se da en determinadas condiciones. En ese sentido, las acciones están relacionadas con los fines y las operaciones a las condiciones, es decir, son los medios con los que se ejecuta una acción. La génesis de la acción reside en las relaciones con la actividad, ya la operación reside en la transformación de la acción que pasa a ser automatizada y utilizada como condición de ejecución de otra acción (LEONTIEV, 1978).

Los motivos, a su vez, pueden ser comprensibles o eficaces. Los primeros son aquellos en que el individuo comprende y reconoce la importancia de la actividad, sin embargo, no son suficientes para impulsar las acciones. Ya los eficaces son aquellos que impulsan las acciones. En el entendimiento de Leontiev (1978; 2004), los motivos comprensibles pueden llegar a ser eficaces y viceversa. En este sentido, defendemos que las tareas pedagógicas proporcionadas en la actividad de estudio deben ser creadoras de motivos eficaces, sea creando nuevos motivos, sea transformando los motivos comprensibles en eficaces.

Por ejemplo, un estudiante que necesita realizar una evaluación, pero se distrae con facilidad, sale para pasear dejando para después la actividad. Demuestra, con ello, que su motivo es apenas comprensible y no eficaz suficiente para sostener su atención en el estudio. Sin embargo, aprender y / o conquistar una buena nota en la evaluación puede convertirse en un motivo eficaz, transformándose entonces en nueva objetivación y, consecuentemente, una nueva actividad motivada que lo impulsa a estudiar.

Las relaciones entre el motivo y el fin de la actividad son mediatizadas por los conceptos de sentidos y significados/significaciones ${ }^{\mathrm{iii}}$. Estos, son considerados por Leontiev (2004) como los principales componentes de la estructura de la conciencia humana. Según el autor, "la significación es la generalización de la realidad que es cristalizada y fijada en un vector sensible, ordinariamente la palabra o la locución” (LEONTIEV, 2004, p. 100).

Las significaciones son siempre sociales, son objetivas, son resultados de actividad humana $\mathrm{y}$, por eso, producidas histórica y socialmente. Se incluyen en ellas los conocimientos científicos, filosóficos y las artes. Ellas llegan al reflejo consciente (pensamiento) por el lenguaje, por la apropiación de conocimientos producidos y reflejados por la humanidad históricamente. Al leer un libro, por ejemplo, no es el libro que entra en nuestra conciencia y sí sus significados expresados por otros individuos que ya los reflejaron y los registraron utilizando lenguaje (conceptos).

La significación está presente objetivamente, es el reflejo de la realidad independiente de la relación subjetiva que el individuo en particular pueda tener con ella. Lo que esa 
determinada significación representa para el individuo faz parte de otro concepto, los sentidos. Leontiev (1978) habla inicialmente en significado objetivo para el sujeto, luego justifica que para evitar la duplicación de términos llamará el último de sentidos.

Sentidos y significados son dos conceptos que están ligados, que se interrelacionan, ya que los sentidos se manifiestan en las significaciones. Los sentidos son personales y se forman a partir de la apropiación de significados. El reflejo consciente o racional depende del sentido subjetivo y personal que las significaciones tengan para el individuo.

En la actividad humana, Leontiev (2004) subraya que la correlación entre las acciones y operaciones objetivas para alcanzar un fin se da por el sentido racional, consciente dado por el individuo durante la actividad. Entonces, “[...] el sentido consciente traduce la relación del motivo al fin" (LEONTIEV, 2004, p. 103), a partir de los "fenómenos objetivos concientizados" (LEONTIEV, 2004, p. 105). En esa dirección, sentidos y significados son determinados por la consciencia social. De manera amplia, tal consciencia está formada a partir de la práctica social del individuo, no sólo en la escuela, sin embargo, la escuela al cumplir su función social de posibilitadora de acceso a conocimientos colabora para el desarrollo psíquico de los estudiantes.

Reside ahí la importancia del trabajo del profesor en el curso de la actividad de estudio para que proponga tareas pedagógicas que favorezcan nuevos motivos y sentidos que sean eficaces y que posibiliten el desarrollo del pensamiento teórico.

Asbahr (2011), en su tesis doctoral, investigó cómo los estudiantes de una cuarta serie de la Enseñanza Fundamental de una escuela pública de São Paulo / Brasil atribuían sentido a la actividad de estudio. Ella descubrió que los estudiantes tenían una enorme necesidad de aprender, ellos querían aprender cosas relacionadas con los contenidos propuestos por la profesora, per se le pedía que realizara muchas copias de la pizarra para el cuaderno (forma mecánica) y, con ello, se volvían acciones vacías de sentido y los motivos que eran apenas comprensibles no eran eficaces.

La autora cita el ejemplo de un niño que ya había repetido la cuarta serie por tres veces y que obtuvo una transformación en el segundo semestre del año escolar. El estudiante tenía un historial de rechazos con relación a las actividades propuestas por la profesora, fue advertido que podría reprochar nuevamente. Al sumado a la posibilidad de reprobación, el niño rompió el brazo y no podía escribir, a profesora pidió entonces que tuviera el acompañamiento de la investigadora, la Asbahr. Ella, a su vez, sugirió al alumno que se realizaran lecturas de poemas. A él le gustó las rimas, percibidas a partir de la estrategia de lectura utilizada por la investigadora y se interesó en leer más poemas, después pidió su ayuda para escribir una carta de amor usando los poemas leídos. Asbahr (2011) concluye que la lectura tenía poco sentido para el estudiante porque no conseguía comprender derecho lo que leía.

Saviani (2011) alerta que la actividad pedagógica necesita considerar dos aspectos fundamentales: la selección de los contenidos a ser enseñados y las formas adecuadas para ello. Con relación al primero, defiende la enseñanza de los clásicos, o sea, de aquellos contenidos que se han firmado históricamente como fundamentales, como esenciales. Tales 
contenidos deben ser estudiados en su dinámica y en relación constante con la actualidad. En cuanto a las formas, trata de la "organización de los medios (contenidos, espacio, tiempo y procedimientos) a través de los cuales, progresivamente, cada individuo singular realice, en la forma de segunda naturaleza, la humanidad producida históricamente" (SAVIANI, 2011, p. $13)$.

Las formas como son organizadas por el profesor las tareas escolares son de esencial importancia para la formación de motivos impulsores de la actividad de estudio. Al enseñar un cálculo, por ejemplo, si el profesor presenta sólo su forma mecánica de resolución, el estudiante puede no tener sentido para esa tarea. Por otro lado, si enseña los conceptos del cálculo y su lógica, favorecerá su comprensión y utilización en diversas otras situaciones de vida, no sólo escolar.

Por eso, si el profesor se centra en actividades simples, que no compulsa al estudiante a realizar un mínimo de esfuerzo, no se le da sentido a la actividad de estudio. Sino que la realiza de manera mecánica como un autómata y sin comprender el contenido ni el significado de la actividad que realizan. Esto os lleva a perder la motivación por estudiar y a no esforzarse en este empeño. En realidad, realizan una acción de estudio, no una actividad de estudio y de lo que se trataría, es entonces el momento de convertir el acto en actividad.

Por tanto, para que los estudiantes se motivan en la realización de su actividad de estudio es necesario que le encuentren significado y sentido personal a la actividad de estudio que realizan. Que debe tener un momento inicial en el que se realiza el análisis y la abstracción iniciales a partir de la observación del objeto de estudio, lo que les permita extraer los datos, la información requerida y necesaria que deberá ser procesada para seguir el método de lo abstracto a lo concreto pensado cumpliendo con las siguientes acciones de la tarea de estudio:

1. Transformación de los datos del problema: Esto implica realizar el análisis esencial para determinar las propiedades (características esenciales del objeto), extraer la conexión esencial entre las características esenciales del objeto de estudio con el fin de separar la relación que constituye la base genética esencial, la célula constituyente.

2. Modelación de dicha relación: En el modelo se establecen los componentes y relaciones esenciales entre las propiedades del objeto de estudio, de manera que se puedan hacer una representación y construir una imagen adecuada del mismo.

3. Transformación del modelo: Con el objetivo de estudiar la relación de importancia sustancial en el proceso general de asimilación de los conocimientos teóricos por cuanto permita comprender la especificidad de la orientación en un plano peculiar. Poder aplicar y adaptar el modelo a las situaciones y casos particulares y singulares en concreto, manifestándose de esta manera lo único en lo diverso.

De esta manera con este esfuerzo intelectual se garantiza su motivación y la movilización por ende de todas las energías de su personalidad en post de alcanzar las metas de la enseñanza propiciando una construcción con sentido y significado con un aprendizaje consciente y desarrollador.

Davidov (1978), Davidov y Slobódchikov (1991) se dedicó a la actividad de estudio. 
Para él la enseñanza de calidad promueve el desarrollo psíquico y la capacidad de pensar por conceptos (pensamiento teórico). Libâneo hace un análisis de los estudios de Davidov y concluye:

\begin{abstract}
En la base del pensamiento de Davydov está la idea de Vygotsky de que el aprendizaje y la enseñanza son formas universales de desarrollo mental. La enseñanza propicia la apropiación de la cultura y el desarrollo del pensamiento, dos procesos articulados entre sí, formando una unidad. Podemos expresar esta idea de dos maneras: a) mientras que el alumno forma conceptos científicos, incorpora procesos de pensamiento y viceversa; b) como forma el pensamiento teórico, desarrolla acciones mentales, mediante la solución de problemas que suscita la actividad mental del alumno. Con ello, el alumno asimila el conocimiento teórico y capacidades y habilidades relacionadas con ese conocimiento (LIBÂNEO, 2004, p. 14).
\end{abstract}

Por ello es recomendable que los profesores logren la conformación de situaciones de enseñanza y aprendizaje que propicien la conformación y la construcción en ellos de bases orientadoras de la acción (BOA) que propicien la construcción de su conocimiento y su desarrollo. Para ello debe romperse con la enseñanza tradicional donde el profesor juega el papel activo para que se propicien situaciones donde el estudiante tome su rol activo y se le dé al estudiante el espacio de tiempo para que pueda pensar, expresar sus ideas, y proponga sus soluciones a los problemas que él mismo elabore y le proponga el profesor.

Sin embargo, cuando se logra que el estudiante le encuentre sentido personal y significado a su actividad de estudio, y se cumplen las tareas de estudio, como las plantea Davidov (1978), se crean las condiciones y premisas para que el estudiante desarrolle su pensamiento teórico, superando su tendencia al pensamiento empírico. Pensamiento que permita al estudiante orientarse en la búsqueda de las esencias de un determinado objeto de estudio, en el trabajo con los conceptos, en el establecimiento de las relaciones esenciales de lo que aprenden, en la deducción de las leyes que rigen el movimiento, su desarrollo. Esto les posibilita la comprensión de mundo en que viven.

Acerca del pensamiento teórico se pueden plantear algunas ideas básicas, que justifican su necesidad en la formación del individuo y la importancia de propiciar su desarrollo desde las primeras edades de la enseñanza escolar de manera que se supere el pensamiento empírico en la configuración de sus sentidos y significados sobre lo que aprenden.

El pensamiento teórico es de trascendental importancia porque se contrapone al pensamiento empírico, propio de niño, el cual permanece en el nivel de las apariencias y en las representaciones simplistas porque "se basa en el principio de generalidad puramente formal y abstracta, pues trabaja con los datos directos de la realidad y en la realidad" (DAVIDOV, 1978, p. 199), y por eso la percibe fragmentadas, inconexa, por lo que ve las propiedades aisladas del objeto concreto.

Mientras que en contraste, el pensamiento teórico trasciende al pensamiento empírico porque parte de la génesis, de las premisas, de las condiciones, busca develar lo esencial, por medio de la abstracción esencial y deducir las leyes y principios más profundos que rigen el desenvolvimiento del objeto, define y se produce en conceptos por medio de la síntesis 
esencial, estableciendo las relaciones, las conexiones internas esenciales entre las propiedades esenciales del objeto de estudio y su desarrollo, viéndolo interconectado con los demás objetos una vez que encuentra lo único en lo diverso por el camino de la ascensión de lo abstracto a lo concreto (DAVIDOV, 1978).

Conociendo del objeto de estudio sus propiedades y las relaciones esenciales entre estas, lo que se constituye en una propiedad en sí misma de dicho objeto, así como sus contradicciones internas; el pensamiento teórico logra, según Davidov (1978), reflejar el fenómeno en su desarrollo, en su evolución, y no sólo en su aspecto directo y externo que es a lo que se dirige el pensamiento empírico. De esta manera se logra la comprensión, la predicción, la aplicación a la vida cotidiana de los principios y leyes que lo rigen en la solución de problemas científicos y técnicos de la sociedad.

Solo el pensamiento teórico es el que le posibilita al hombre orientarse y acercarse cada vez más a la esencia de las cosas, de manera que le permite develar progresivamente las condiciones y contradicciones que engendran el objeto de estudio. Para determinar los atributos de lo que se pretende definir por medio de los conceptos y las categorías, que no son otra cosa que el reflejo de las esencias y las relaciones esenciales del objeto de estudio hasta el nivel de profundidad que le sea posible al hombre en un momento histórico concreto.

En estudios realizados por Davidov y sus seguidores se ha demostrado que es posible la conformación y el desarrollo del pensamiento teórico de los estudiantes desde las edades más tempranas, solo hay que crear las condiciones que lo propicien como son la conformación de una situación de enseñanza aprendizaje que condicione el aprendizaje activo, la problematización de las situaciones de enseñanza y aprendizaje.

La importancia del pensamiento teórico (dialéctico y complejo por naturaleza) consiste en que orienta al hombre en la generalización esencial y en el establecimiento de las relaciones esenciales entre las esencias en el proceso de construcción y elaboración de los conceptos por el método de la ascensión de lo abstracto a lo concreto pensado, de manera que durante este proceso de integración, junto con las condiciones y contradicciones que le dan origen, se van realizando acciones que logran transformar el objeto y el reflejo que de este se tiene.

Por lo planteado hasta este momento nos proponemos realizar un conjunto de recomendaciones que contribuyan a la formación del significado y el sentido personal de la actividad de estudio y de cómo, cumpliendo con las tareas de estudio planteadas por Davidov (1978), se contribuye en la conformación y desarrollo del pensamiento teórico de los estudiantes.

Las recomendaciones a los profesores, todas con base en los aportes teóricos de la perspectiva histórico-cultural, giran en torno da relación entre contenido y forma. Algunas ya habían sido señaladas en el transcurso del texto, las rescatamos y presentamos junto con otras, a saber:

1. Crear situaciones de enseñanza y aprendizaje donde se propicie el aprendizaje activo por parte de los estudiantes, donde se problematice la realidad de estudio. Estar muy atento a las palabras espontáneas de los estudiantes y dar oportunidades para que expresen 
sus comprensiones y necesidades (significaciones), porque los sentidos se expresan en las significaciones;

2. Darles también una mayor participación a los estudiantes en la elaboración de problemas y en la elaboración de las estrategias y vías de la búsqueda de las soluciones;

3. Darle tempo y espacio a los estudiantes para que piensen, para que reflexionen y la oportunidad de que cometan errores sin temas a la equivocación;

4. Evitar las evaluaciones y la estigmatización del estudiante por su error;

5. Promover el aprendizaje de los conceptos a partir de un hecho y desde este conducir las acciones del pensamiento hasta develar su esencia, de manera a criar en ele motivos eficaces para un aprendizaje con sentido;

6. Posibilitar que el estudiante tenga conocimientos y información de la importancia y el significado de lo que aprende para su vida diaria personal pasada, presente y futura, para la ciencia, la técnica y para la sociedad en su conjunto;

7. No darles a los estudiantes los conocimientos ni los contenidos de su aprendizaje de manera totalmente elaborada, acabada, ya hecha, sino propiciar las condiciones para que con distintos niveles de ayuda pueda elaborarlo por sí mismo y en interacción con el otro igual. Sin embargo, si abstenerse de su trabajo de enseñar, la ayuda de los profesores con explicaciones, aclaraciones y cuestionamientos críticos debe hacerse presente;

8. Tener en cuenta las dinámicas grupales y propicias que se creen zonas de desarrollo eminentes que lo impulsen y lo halen al desarrollo.

A partir de la base teórica adoptada defendemos el trabajo del profesor como de fundamental importancia, ya que es él quien dirige el proceso de enseñanza y aprendizaje, es él quien tiene el más alto grado de conocimiento acerca del contenido de la clase, es él quien precisa ser creativo al planear formas de envolver los estudiantes en la actividad de estudio y es él quien va a crear oportunidades para que esos estudiantes sean sujetos activos y que puedan realizar intentos, puedan errar, puedan utilizar los conceptos ya interiorizados para el análisis de situaciones generales de la vida cotidiana y escolar, entre tantas otras posibilidades.

Destacamos que se trata de indicativos para auxiliar en la reflexión de los profesores al definir las tareas escolares. No tenemos un recetario listo y acabado, ni siquiera creemos que exista. Nuestros esfuerzos son de constantes aproximaciones con los conceptos de sentidos y de sus influencias en la ejecución de las tareas escolares para posibilitar el desarrollo del pensamiento teórico.

No podemos dejar de destacar que adoptamos como base de sustentación del presente trabajo a los autores clásicos (Leontiev y Davidov), pero en la actualidad diversos investigadores han contribuido efectivamente en las investigaciones de la teoría históricocultural y de la actividad de estudio en todo el mundo, el nivel de información para futuras investigaciones apuntamos sólo algunos en Brasil y en Cuba.

En Brasil podemos citar, principalmente, autores ligados a la Pedagogia histórico- 
crítica como Saviani (2011), Duarte (2013), Martins (2013), y autores ligados a la Enseñanza desarrollista como los integrantes del "Grupo de Estudos e Pesquisas em Didática Desenvolvimental e Profissionalização Docente (GEPEDI)" e "Grupo de Estudos e Pesquisa da Atividade Pedagógica (GEPAPe)". En Cuba podemos citar, entre otros, Beatón (2013) y Serra (2017). Además, tenemos un investigador originario de Cuba y que vivió la última etapa de su vida en Brasil, el Gonzáles Rey (1949-2019).

\section{Conclusiones}

En el momento histórico en que nos encontramos, si se quiere que nuestros estudiantes sean ciudadanos activos y transformadores de la sociedad, que floten sobre su tempo y sean seres con alto nivel de desarrollo, debemos propiciar la formación en ellos de significados y sentidos personales de los contenidos de sus actividades de estudio, de manera que puedan desarrollar su pensamiento teórico que les permita develar y llegar a las esencias de aquello que constituya objeto de su estudio.

Iniciamos el texto proponiéndonos a investigar el papel de los sentidos para la actividad de estudio específicamente en las tareas propuestas por los profesores con el propósito de desarrollar el pensamiento teórico de sus estudiantes y podemos decir que la motivación y los sentidos personales sobre la actividad de estudio determinan la conducta, el interese y el rendimiento, así como el grado de consciencia de los estudiantes durante el aprendizaje de determinado contenido en lo proceso de construcción de sus conocimientos.

Los profesores deben tener conocimientos mínimos sobre los motivos y los sentidos de la actividad de estudio de sus estudiantes en la planificación y la organización de su proceso docente educativo con el fin de lograr aprendizajes conscientes y desarrolladoras. De esta forma, se pueden crear las condiciones para la transformación de motivos comprensibles en motivos eficaces, aquellos que tienen sentido para los estudiantes y impulsan su actividad de estudio.

El profesor tiene un papel central en el proceso de enseñanza y aprendizaje, corresponde a él conocer los contenidos esenciales a ser estudiados y las formas para hacerlo. Las tareas pedagógicas propuestas deben tener en cuenta al estudiante real, necesitan comprenderlo como un sujeto activo que se apropia de las significaciones sociales y las analiza para crear sus motivaciones y dar sentido a las actividades de las cuales participa.

Las tareas educativas, también pueden crear motivos generales y significativos que, además de incitar la acción, pueden dar un sentido determinado a lo que el estudiante hace. Otra tarea importante es la de hacer que los estudiantes tengan conciencia de sus motivos, pues con ello pueden adquirir una visión más lúcida y eficaz de los estudios y adquirir nuevas conductas.

La escuela necesita proporcionar el acceso a los conocimientos y conceptos producidos por la humanidad históricamente y crear condiciones para que el estudiante produzca nuevos conocimientos a partir de los ya elaborados. En este proceso el alumno desarrolla sus funciones psíquicas superiores, lo que genera las condiciones para pensamiento 
teórico capaz de pensar por conceptos, capaz de analizar teóricamente la práctica social como un todo.

Las recomendaciones aquí lanzadas, no pueden ser comprendidas de manera mecánica y lineal, son sólo algunos indicativos que pueden ser considerados puntos de apoyo para reflexiones junto con los profesores, tomando como base las condiciones de su realidad concreta. No pretendemos aquí agotar el asunto, sino reforzar la necesidad de reflejar la actividad de estudio y, en especial, la función del profesor al proponer tareas pedagógicas.

\section{Referencias}

ASBAHR, Flávia da Silva Ferreira. "Por que aprender isso, professora?" Sentido pessoal e atividade de estudo na Psicologia Histórico-Cultural. 2011. 220 f. Tese (Doutorado em Psicologia Escolar e do Desenvolvimento Humano) - Instituto de Psicologia, Universidade de São Paulo, São Paulo, 2011. Doi: 10.11606/T.47.2011.tde-24032011-094830.

BEATÓN, Guillermo Arias. La Psicología Social y lo histórico cultural. In: COLECTIVO DE AUTORES. Psicodrama en Cuba: experiencias, vivencias, sentido. 1. ed. La Habana: Caminos, 2013.

DAVÍDOV, Vasili Vasilovich; SLOBÓDCHIKOV, Vladimir. La enseñanza que desarrolla en la escuela del desarrollo. In: MÚDRIK, A. B. (ed.). La educación y la enseñanza: una mirada al futuro. Moscú: Progreso, 1991. p.118-144.

DAVIDOV, Vasili Vasilovich. Tipos de generalización en la enseñanza. La Habana, Cuba: Pueblo y Educación, 1978.

DUARTE, Newton. Vigotski e a pedagogia histórico-crítica: a questão do desenvolvimento psíquico. Nuances: estudos sobre Educação, Presidente Prudente, SP, v. 24, n. 1, p. 19-29, jan./abr. 2013. http://dx.doi.org/10.14572/nuances.v24i1.2150.

LEONTIEV, Alexis Nikolaevich. Actividad, Conciencia y Personalidad. Buenos Aires, Argentina: Ciencias del Hombre, 1978.

LEONTIEV, Alexis Nikolaevich. Desenvolvimento do Psiquismo. São Paulo: Moraes, 2004.

LIBÂNEO, José Carlos. A didática e a aprendizagem do pensar e do aprender: a Teoria Histórico-cultural da Atividade e a contribuição de Vasili Davydov. Revista Brasileira de Educação, Rio de Janeiro, n. 27, p. 5-24, set./dez. 2004. http://dx.doi.org/10.1590/S141324782004000300002.

MARTINS, Lígia Márcia. O desenvolvimento do psiquismo e a educação escolar: contribuições à luz da Psicologia Histórico-Cultural e da Pedagogia Histórico-Crítica. Campinas: Autores Associados, 2013.

SAVIANI, Dermeval. Pedagogia histórico-crítica: primeiras aproximações. 11. ed. rev. Campinas: Autores Associados, 2011. 
SERRA, Diego Jorge González. Los niveles de la motivación: Teoría y evaluación. La Habana, Cuba: Pueblo y Educación, 2017.

\section{Notas}

${ }^{i}$ A. N. Leontiev (1903-1979), psicólogo soviético, participó del equipo inicial de Vigotski junto con Luria, participó de la primera generación de la escuela psicológica. Fue proponente de la Teoría de la Actividad.

ii V. V. Davidov (1930-1998), psicólogo soviético, fue de la tercera generación de la escuela psicológica, partió de los estudios de Vigotski y Leontiev para crear su propia versión de la Teoría de la Actividad, se profundizó en la actividad de estudio y la enseñanza desarrollista.

iii En los textos estudiados, aparecen las palabras "significados" y "significaciones" como sinónimos. Ambas formas se utilizarán en el curso del presente artículo en el entendido de que sólo son variaciones de traducción. 